SNP array for copy number variations (CNV's) showed a unique $550 \mathrm{~kb}$ duplication involving SOX3, RP1-177G6, and CDR1 genes, and the microRNA MIR320D2. This CNV was absent in 13,839 controls.

Conclusions A SRY negative 46, XX male with renal hypodysplasia was found to have an exceedingly rare duplication involving the SOX-3 gene, proving its role in sex determination and suggesting its evolvement in kidney development.

\section{PETERS PLUS SYNDROME}

doi:10.1136/archdischild-2012-302724.0741

I Maaloul, L Sfaihi, K Baklouti, S Ben Ameur, H Aloulou, T Kamoun, M Hachicha. Hedi Chaker Hospital, Sfax, Tunisia

Background Peters Plus syndrome is an autosomal recessive and rare disorder characterized by a variety of anterior eye chamber defects, of which the Peters anomaly occurs most frequently. Other major symptoms are a disproportionate short stature, developmental delay, characteristic craniofacial features, and cleft lip and/or palate.

Observations We report 4 cases of peters plus syndrome who were admitted in the department of pediatric of sfax. The family history revealed consanguineous parents in 3 cases, ocular abnormalities in 2 cases and the death of sibling in one case. Multiples abnormalities were noted in all cases just after birth like bilateral corneal opacities and facial dysmophism. 3 new borns had Skeletal system abnormalities (Short limbs, Short, broad hands, clinodactyly). Congenital heart malformations were present in 2 cases and renal anomalies were noted in one case. During the evolution, 3 patients presents a failure to thrive and developmental delay. One patient was died because of pneumonia.

Conclusion Peters plus syndrome is inherited in an autosomal recessive manner. The diagnosis is based on clinical findings and genetic analysis; prenatal diagnosis for pregnancies at increased risk are possible if the disease-causing mutations in the family are known.

\section{CHROMOSOMAL ABNORMALITIES IN A TERTIARY NEONATAL INTENSIVE CARE UNIT}

doi:10.1136/archdischild-2012-302724.0742

'A Dursun, ${ }^{2} \mathrm{~T}$ Tos, 'N Hakan, 'BS Karagol, 'N Karadag, 'B Aydin, 'A Zenciroglu, 'S Beken, 'N Okumus. 'Neonatology; 'enetic, Dr Sami Ulus Maternity and Children's Health and Diseases Training and Research Hospital, Ankara, Turkey

Background and Aim Congenital malformations and chromosomal abnormalities are important problems for the neonatal morbidity and mortality especially in well developed countries. In this study we investigated the prevalence and distribution of the chromosomal abnormalities in our NICU.

Method Chromosomal abnormalities identified between 2008 and 2012 were retrospectively analyzed. Also, demographic features and concomitant congenital malformations were also collected. Cytogenetic analysis was performed on peripheral blood of newborns by standard chromosomal analysis methods. According to our hospital protocols, newborns with more than one major malformation or with 1 major plus 2 minor abnormalities were scheduled for chromosomal abnormalities.

Results During the study period, 431 chromosomal analyses (3.2\%) were performed among the 5170 hospitalized newborns. $78.4 \%$ of the cases had normal chromosomal karyotype. Consanguinity rate was $27.9 \%$. Major chromosomal abnormality rate was $1.8 \%$.

Frequency of chromosomal abnormalities were as follows; Trisomy 21 13.5\% $(n=58)$, trisomy 18 2.3\% $(n=10)$, trisomy $131.2 \%$ $(n=5), 45 \mathrm{XO} 0.5 \%(n=2)$ and other chromosomal abnormalities were $4.2 \%(n=18)$. Congenital heart abnormalities (85.9\%), craniofascial abnormalities (44.6\%) and genito-urinary anomalies (16.9\%) were most common concomittant malformations. Cardiovascular abnormalities were most common malformations in newborns with trisomy 21.

Conclusion Frequency and distribution of the chromosomal abnormalities in our NICU were similar compering with other populational studies. Trisomy 21 was most common chromosomal abnormality. Newborns with malformations in more than two organ system should be investigated chromosomally as well.

\section{LYSOSOMAL STORAGE DISORDERS IN NON- IMMUNOLOGICAL HYDROPS FETALIS - MORE COMMON THAN ASSUMED?}

doi:10.1136/archdischild-2012-302724.0743

${ }^{1} \mathrm{C}$ Whybra, ${ }^{2} \mathrm{E}$ Mengel, ${ }^{3} \mathrm{~F}$ Bahlmann, ${ }^{4} \mathrm{C}$ Kampmann, ${ }^{2} \mathrm{M}$ Beck, ${ }^{1} \mathrm{E}$ Mildenberger. ${ }^{1}$ Neonatology; ' 2 Department of Lysosomal Storage Disorder, Villa Metabolica, University Medical Center of the Johannes Gutenberg University, Mainz; ${ }^{3}$ Department of Obstetrics and Gynecology, Buergerhospital, Frankfurt/Main; ${ }^{4}$ Department of Pediatric Cardiology, Medical Center of the Johannes Gutenberg University, Mainz, Germany

Background Although non immunological hydrops fetalis (NIHF) is a very rare disorder, the disturbance accounts for a disproportionate share $(3 \%)$ of overall mortality in the perinatal period. Lysosomal storage disorders (LSD) are only exceptionally considered to be the cause of NIHF. The reported incidence is about $1 \%$. On the other hand, in about $18 \%$ of all cases, NIHF is classified as idiopathic.

Patients and methods We report four cases of transient NIHF due to LSD and reviewed the literature for LSD associated with NIHF.

Results At present, 12 different LSD are described to be associated with NIHF. The majority of reported patients already had a family history of NIHF, which had not been investigated. A diagnostic approach to the fetus with NIHF due to suspected LSD is suggested.

Conclusions Extensive and thorough investigation of the etiology of NIHF is obligatory. In particular, LSD should be considered in idiopathic NIHF. Enzymatic studies in chorionic villous samples or amniotic cultured cells, once the most common conditions associated with NIHF have been ruled out, should be performed. We assume that the incidence of LSD in NIHF is significantly higher than the estimated $1 \%$ reported in previous studies. This is important for genetic counseling, as there is at first, a high risk of recurrence and, secondly, the availability of enzyme replacement therapy for an increasing number of LSD.

\section{CHOLESTEROL ESTER TRANSFER PROTEIN GENE POLYMORPHISM AND SELECTED LIPIDS PARAMETERS IN CHILDREN FROM FAMILIES WITH HISTORY OF CVS DISEASES}

doi:10.1136/archdischild-2012-302724.0744

E Pac-KoŻuchowska, A Mroczkowska-Juchkiewicz, A Pawłowska-Kamieniak, K Kominek, D Gołyska. Medical University, Lublin, Poland

The human population especially predisposed to early development of atherosclerosis are children from families with history of cardiovascular system diseases. The aim of this study was to examine lipids parameters associated with cardiovascular diseases and polymorphisms of G279A located in the Cholesterol Ester Transfer Protein (CETP) gene.

Material/methods The study covered 30 children aged 5-6 years from families with history of cardiovascular system diseases. The children were examined physically, and nutritional status assessed. In all of the children examined, the blood concentrations of 